\title{
Experimental Investigation of TDD Reciprocity-Based Zero-Forcing Transmit Precoding
}

\author{
Per Zetterberg \\ ACCESS Linnaeus Center, KTH Royal Institute of Technology, Osquldasväg 10, 10044 Stockholm, Sweden \\ Correspondence should be addressed to Per Zetterberg, per.zetterberg@ee.kth.se
}

Received 2 June 2010; Revised 16 November 2010; Accepted 14 December 2010

Academic Editor: Dragan Samardzija

Copyright ( $) 2011$ Per Zetterberg. This is an open access article distributed under the Creative Commons Attribution License, which permits unrestricted use, distribution, and reproduction in any medium, provided the original work is properly cited.

We describe an implementation of TDD reciprocity based zero-forcing linear precoding on a wireless testbed. A calibration technique which self-calibrates the base-station without the need for help from other nodes is described. Performance results in terms of downlink channel estimation error as well as bit error rate (BER) and signal to interference noise and distortion ratio (SINDR) are presented for a scenario with two base-stations and two mobile stations, with two antennas at the base-stations and a single antenna at the mobile-station. The results show considerable performance improvements over reference schemes (such as maximum ratio transmission). However, our analysis also reveals that the hardware impairments significantly limit the performance achieved. We further investigate how to model these impairments and attempt to predict the SINDR, such as what would be needed in a coordinated multipoint (CoMP) scenario where scheduling is performed jointly over the two cells. Although the results are obtained for a MISO scenario the general conclusions are relevant also for MIMO scenarios.

\section{Introduction}

Multiple antenna systems (MASs) are widely employed to enhance the performance of wireless communication systems. Many techniques using MAS, in particular those that address interference issues, require extensive channel knowledge at the transmitter [1-3]. One way of accessing this information is to utilise the reciprocity principle which states that the channel between two antennas is the same in both directions (i.e., irrespectively of which antenna is used as transmitter and which is used as receiver) [4]. This property holds only if the carrier frequency used in both directions is the same, and therefore only time division duplex (TDD) systems can make use of this principle. Thus by designing a system so that a base-station is able to first receive signals from a number of mobiles in the uplink, it may estimate the channel of those mobiles and later utilise this channel information to enhance the signal at a targeted mobile while minimising the interference generated at the (victim) stations when transmitting in the downlink. The required uplink signals will in some cases be available because the mobiles need to send uplink payload data, and therefore the channel information is obtained more or less "for free." However while the channel is reciprocal, the hardware is not. Calibration procedures have to be employed to account for this.

The principles for TDD-based precoding have been known for a long time (see, e.g., [5]) although practical aspects of the technique have received relatively little attention in the literature. However, a few papers exist; see for example [6-9]. Addressing the issue is timely considering the current interest in multicell cooperation with interference suppression.

Paper [7] investigates the impact of phase, frequency, and delay errors on the performance of a single MIMO link. However, the transmitter is not trying to suppress interchannel interference which makes the system quite insensitive to the errors.

Paper [8] proposes a calibration technique whereby the two ends of a link estimate the impulse response between them (a matrix of impulse responses in the MIMO case). The receiver encodes and feeds back its impulse response, so that the transmitter is able to compute compensation matrices. The two measurements of the channel needed to calculate 
the compensation matrix have to be performed within the channel coherence time. The paper also presents estimate of compensation filters estimated from experimental data in the SISO case. Paper [6] uses a similar technique. The performance of the channel estimation in [6] seems to be similar to that obtained herein.

Paper [9] introduces a calibration technique whereby a base- or mobile-station can calibrate itself without the assistance of another entity (such as another base- or mobile-station). The technique is based on sending signals between the transmitters and receivers internally in the base-station and thereby obtaining the required calibration parameters. The calibration signals are routed using couplers and switches. The paper presents measurements in terms of amplitude and phase errors and antenna diagrams.

This paper uses a modified version of the technique of [9]. The difference is that in our implementation the calibration signals are sent over the antennas eliminating the need for additional circuitry and the inaccuracies that these components may introduce. On the other hand, our implementation requires an interrupt in the transmission while the solution in [9] enables concurrent transmission and calibration. We also indicate how to utilise our calibration technique in a MIMO scenario. We further describe the implementation of the calibration and zero-forcing [3] precoding on the universal software radio peripheral (USRP) using RFX1800 daughterboards (see http://www.ettus.com/). The results show considerable performance improvements over reference schemes (such as maximum ratio transmission) in a two-base-station two-mobile scenario. Results in terms of the performance of downlink channel estimation (from uplink data), downlink bit error rate (BER), and signal to interference, noise, and distortion ratio (SINDR) are presented.

An empirical model of the channel prediction performance is fitted to the measurements. However, the channel estimation error is not the only impairment. In addition to this problem there are also distortions due to phasenoise, amplifier nonlinearities, and other sources [10]. In two recent papers on MIMO systems the combined contribution of these distortions has been modeled as spatially white Gaussian noise $[11,12]$. However, neither of these two papers treats interference suppression at the transmitter (as does this paper). In this paper we observe that the distortion significantly degrades the performance of our system. Then we use the distortion model introduced in $[11,12]$ and find reasonable agreement with our results in average. However, the model is not good enough to predict the distortion in a certain timeslot. Such instantaneous information is desirable when performing link adaption (selecting the coding and modulation scheme for a user) in coordinated multipoint (CoMP) scenarios.

The paper is organised as follows. In Section 2 we describe the calibration technique used in the paper. The implementation is described in Section 3 while measurement results are presented in Section 5. In Section 4 we compare measurement result with results obtained through simulations. Finally, the conclusions are summarised in Section 7. Table 1 lists some of the notational conventions used.
TABLE 1: Mathematical notations.

\begin{tabular}{|c|c|}
\hline Notation & Description \\
\hline$s$ & $\begin{array}{l}\text { Lowercase italic letters are real or complex } \\
\text { scalars. }\end{array}$ \\
\hline $\mathbf{v}$ & $\begin{array}{l}\text { Boldface lowercase letters are real or complex } \\
\text { vectors. }\end{array}$ \\
\hline $\mathbf{M}$ & Uppercase boldface letters are matrices. \\
\hline $\mathbf{M}^{c}$ & Complex conjugate of the matrix $\mathbf{M}$. \\
\hline $\mathbf{M}^{T}$ & Transpose of the matrix $\mathbf{M}$. \\
\hline $\mathbf{M}^{*}$ & Complex conjugate transpose of the matrix $\mathbf{M}$. \\
\hline$\|\mathbf{M}\|$ & Frobenius norm of matrix $\mathbf{M}$. \\
\hline $\mathbf{v}_{1} \odot \mathbf{v}_{2}$ & Element-wise multiplication. \\
\hline $\operatorname{diag}(\mathbf{v})$ & $\begin{array}{l}\text { A diagonal matrix with the elements of } \mathbf{v} \text { along } \\
\text { the diagonal. }\end{array}$ \\
\hline $\operatorname{diag}\left(c_{1}, \ldots, c_{m}\right)$ & $\begin{array}{l}\text { A diagonal matrix with scalars } c_{1}, \ldots, c_{m} \text { along } \\
\text { the diagonal. }\end{array}$ \\
\hline
\end{tabular}

\section{Calibration Procedure}

We consider a downlink scenario where the aim is to obtain (transmitter) channel information at the base-station. The considered situation is depicted in Figure 1. The picture shows a base-station with $m$ antennas and a mobile-station with $n$ antennas. Each transmitter and receiver chain is characterized by an unknown gain and phase. The calibration coefficients are obtained using signals generated and received locally at the base-station. The switches between the receiver/transmitter pairs can be set independently. The effective downlink channel, $\mathbf{H}^{\mathrm{DL}}$ (from base-station to mobile-station), is given by

$$
\mathbf{H}^{\mathrm{DL}}=\mathbf{C}^{\mathrm{MS}, \mathrm{rx}} \mathbf{H C}^{\mathrm{BS}, \mathrm{tx}},
$$

where $\mathrm{C}^{\mathrm{MS} \text {,rx }}$ and $\mathrm{C}^{\mathrm{BS} \text {,tx }}$ are diagonal and contain the complex gain of the corresponding receiver $(=\mathrm{rx}) / \operatorname{transmitter}(=\mathrm{tx})$ chain in the mobile-station(MS) or base-station(BS) along the diagonal.

In the same way the effective uplink channel is given by

$$
\mathbf{H}^{\mathrm{UL}}=\mathbf{C}^{\mathrm{BS}, \mathrm{rx}} \mathbf{H}^{T} \mathbf{C}^{\mathrm{MS}, \mathrm{tx}} \text {. }
$$

In the following, we propose a technique to obtain a matrix $\widetilde{\mathbf{H}}$ which has the same row-space as the true downlink channel $\mathbf{H}^{\mathrm{DL}}$. This information is sufficient for zero-forcing techniques such as in this paper. We define the matrix $\tilde{\mathbf{H}}$ as

$$
\tilde{\mathbf{H}}=\mathbf{H}^{\mathrm{UL}, T} \operatorname{diag}\left(1, \frac{c_{2}^{\mathrm{BS}, \mathrm{tx}} c_{1}^{\mathrm{BS}, \mathrm{rx}}}{c_{1}^{\mathrm{BS}, \mathrm{tx}} c_{2}^{\mathrm{BS}, \mathrm{rx}}}, \ldots, \frac{c_{m}^{\mathrm{BS}, \mathrm{tx}} c_{1}^{\mathrm{BS}, \mathrm{rx}}}{c_{1}^{\mathrm{BS}, \mathrm{tx}} c_{m}^{\mathrm{BS}, \mathrm{rx}}}\right) .
$$

We first need to show how the base-station obtains the information needed to calculate $\tilde{\mathbf{H}}$. The uplink channel matrix can obviously be obtained from the uplink signals. Next, the elements of the diagonal matrix can be obtained by the following calibration procedure. By sending a signal from antenna no. 1 to antenna no. 2, the base-station obtains $c_{1}^{\mathrm{BS}, \mathrm{tx}} c_{2}^{\mathrm{BS} \text {,rx }} c$, where $c$ is the coupling between the antennas. Likewise it may estimate the channel from antenna no. 2 to 


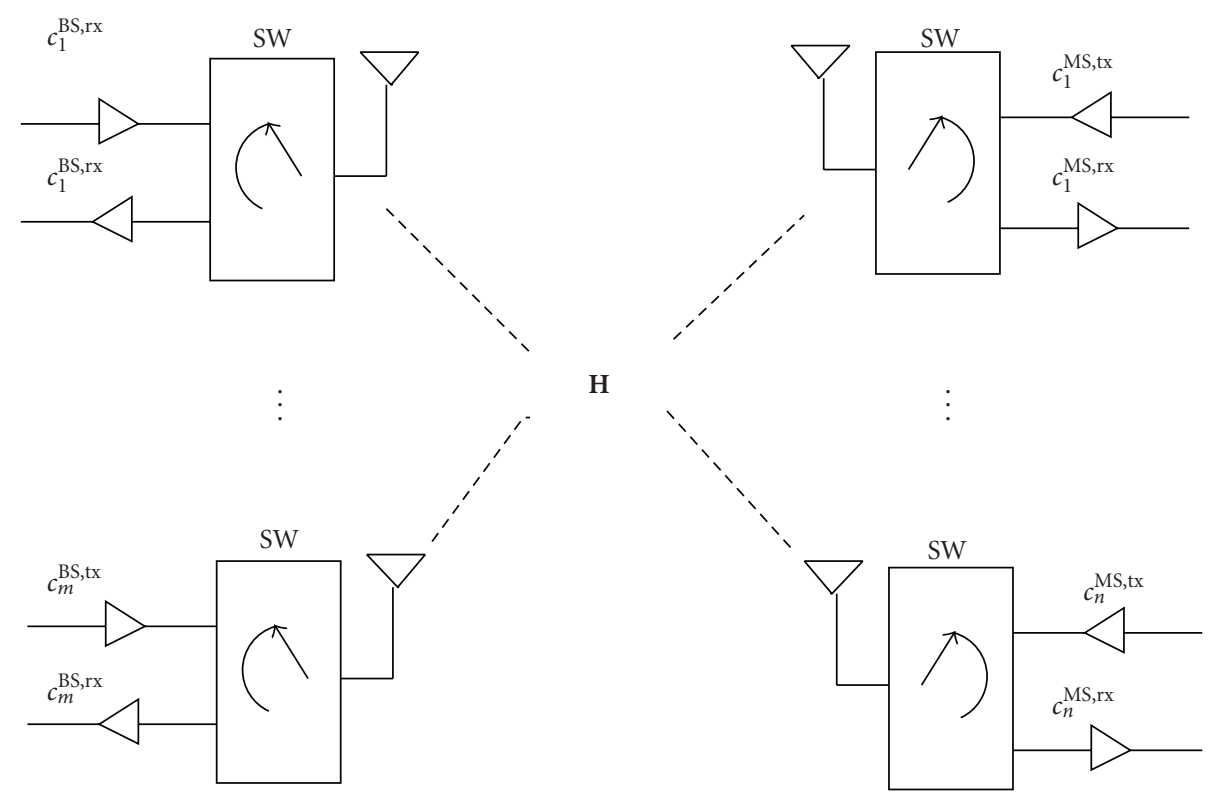

FIgURE 1: Illustration of calibration procedure.

antenna no. 1 by transmitting in the opposite direction, thus attaining an estimate of $c_{2}^{\mathrm{BS}, \mathrm{tx}} c_{1}^{\mathrm{BS}, \mathrm{rx}} c$. From these two estimates the quotient $c_{2}^{\mathrm{BS}, \mathrm{tx}} c_{1}^{\mathrm{BS}, \mathrm{rx}} / c_{1}^{\mathrm{BS}, \mathrm{tx}} c_{2}^{\mathrm{BS}, \mathrm{rx}}$ is obtained. By repeating this procedure by transmitting signals between element no. 1 and all the other elements in the array (one at a time), all the elements of the diagonal matrix in (3) can be obtained.

The next step is to obtain a relation between the true downlink channel $\mathbf{H}^{\mathrm{DL}}$ and our estimate $\widetilde{\mathbf{H}}$. This is done through the derivations in (4)-(6)

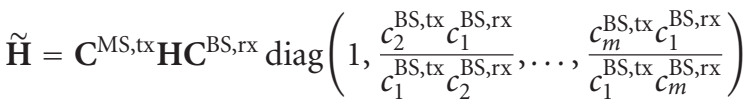

$$
\begin{aligned}
& =\frac{1}{c_{1}^{\mathrm{BS}, \mathrm{tx}}} \mathbf{C}^{\mathrm{MS}, \mathrm{tx}} \mathbf{H C}^{\mathrm{BS}, \mathrm{rx}} \\
& \times \operatorname{diag}\left(c_{1}^{\mathrm{BS}, \mathrm{tx}}, \frac{c_{2}^{\mathrm{BS}, \mathrm{tx}} c_{1}^{\mathrm{BS}, \mathrm{rx}}}{c_{2}^{\mathrm{BS}, \mathrm{rx}}}, \ldots, \frac{c_{m}^{\mathrm{BS}}, \frac{\mathrm{tx}}{1 \mathrm{BS}, \mathrm{rx}}}{c_{m}^{\mathrm{BS}, \mathrm{rx}}}\right) \\
& =\frac{c_{1}^{\mathrm{BS}, \mathrm{rx}}}{c_{1}^{\mathrm{BS}, \mathrm{tx}}} \mathbf{C}^{\mathrm{MS}, \mathrm{tx}} \mathbf{H} \operatorname{diag}\left(1, c_{2}^{\mathrm{BS}, \mathrm{rx}}, \ldots, c_{m}^{\mathrm{BS}, \mathrm{rx}}\right) \\
& \times \operatorname{diag}\left(c_{1}^{\mathrm{BS}, \mathrm{tx}}, \frac{c_{2}^{\mathrm{BS}, \mathrm{tx}}}{c_{2}^{\mathrm{BS}, \mathrm{rx}}}, \ldots, \frac{c_{m}^{\mathrm{BS}, \mathrm{tx}}}{c_{m}^{\mathrm{BS}, \mathrm{rx}}}\right) \\
& =\frac{c_{1}^{\mathrm{BS}, \mathrm{rx}}}{c_{1}^{\mathrm{BS}, \mathrm{tx}}} \mathbf{C}^{\mathrm{MS}, \mathrm{tx}} \mathbf{H} \operatorname{diag}\left(c_{1}^{\mathrm{BS}, \mathrm{tx}}, c_{2}^{\mathrm{BS}, \mathrm{tx}}, \ldots, c_{m}^{\mathrm{BS}, \mathrm{tx}}\right) \\
& =\frac{c_{1}^{\mathrm{BS}, \mathrm{rx}}}{c_{1}^{\mathrm{BS}, \mathrm{tx}}} \mathbf{C}^{\mathrm{MS}, \mathrm{tx}} \mathbf{H C}^{\mathrm{BS}, \mathrm{tx}} \text {. }
\end{aligned}
$$

The estimate (6) obviously differs from the true downlink channel given by (1). Note that $\widetilde{\mathbf{H}}$ and $\mathbf{H}^{\mathrm{DL}}$ are related through

$$
\widetilde{\mathbf{H}}=\frac{c_{1}^{\mathrm{BS}, \mathrm{rx}}}{c_{1}^{\mathrm{BS}, \mathrm{tx}}} \mathbf{C}^{\mathrm{MS}, \mathrm{tx}}\left(\mathbf{C}^{\mathrm{MS}, \mathrm{rx}}\right)^{-1} \mathbf{H}^{\mathrm{DL}} .
$$

When applying zero-forcing, knowing the row-space of $\widetilde{\mathbf{H}}$ is sufficient. It is evident from (7) that this information may be obtained from $\widetilde{\mathbf{H}}$. In cases other than zero-forcing, using $\widetilde{\mathbf{H}}$ in place of the true channel matrix $\mathbf{H}^{\mathrm{DL}}$ is a subject for further study.

In a typical application, the calibration, that is, the transmission between the antenna elements of the basestation would be performed at the rate of change of the gain and phase of the receiver and transmitter hardware. Generally, such changes are attributed to temperature, and thus the changes should be rather slow.

However, the channel coherence time, that is the variability of the propagation channel $\mathbf{H}$, is much faster. In typical cellular and wireless LAN applications with Rayleigh fading typical update times are on the order of milliseconds. Even with those updates rates the channel can change substantially between the time of channel estimation and use. A second source of inaccuracy that should not be forgotten is thermal noise.

A practical issue to consider regarding the selected calibration scheme is that the transmission of the calibration signal can cause interference somewhere else. However, the signal can be made very weak. In fact a significant requirement is that receiver chain is not saturated from an overly strong signal. Another requirement is that the signal actually passes all the way through the transmitter chain, the transmitting antenna, the receiving antenna, and the receive chain and does not leak through.

In the implementation herein we used a calibration signal which was $30 \mathrm{~dB}$ weaker than the signals transmitted from the mobile-station (we are here referring to the power at the transmitter). This allowed us to use the same gain control word setting at the receiver, during calibration as well as during measurements. When this is not the case (i.e., variable gain control word is used) the base-station would need to 


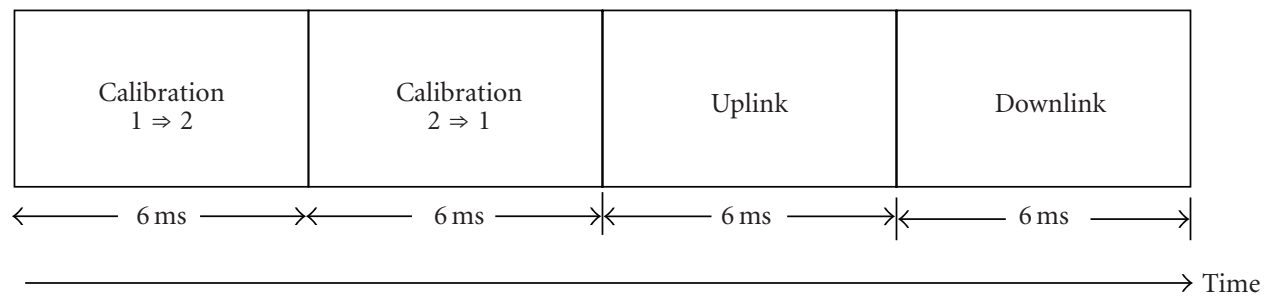

FIGURE 2: The multiframe in the USRP implementation.

create tables of the gain and phase of its receiver chains as a function of the gain control word. The base-station would then use these values to adjust the calibration coefficients accordingly.

\section{Implementation}

Our implementation was done on the universal software radio peripheral (USRP1). This platform consists of a motherboard with a USB interface, an FGPA, a microcontroller, and four $64 \mathrm{MHz}$ ADC and $128 \mathrm{MHz}$ DAC converters, [13]. The board interfaces to a range of transceiver daughterboards for various frequency bands (see http://www.ettus .com/). We are using a pair of RFX1800 daughter-boards on our USPRs. The USRP board is generally connected to a Linux PC which is also the case herein. The GNU Radio project (see http://www.gnuradio.com/) provides a software framework and lots of signal processing modules. In our implementation herein we are however only using the functionality to receive and transmit buffers provided by GNU Radio while all the signal processing is done in Matlab.

We have utilised two nodes, one base-station and one mobile-station, and used emulation techniques to investigate a system consisting of two base-stations and two mobilestations, as will be described in more detail below.

The node representing the base-station is employing two antennas, and the mobile-station is using a single antenna. We are using an OFDM modulation with a sample frequency of $2 \mathrm{MHz}$. An FFT length of eight with a cyclic prefix length of two samples is employed, resulting in a subcarrier spacing of $250 \mathrm{kHz}$. Of the eight subcarriers the innermost five are used while the remaining three are nulled. The modulation scheme used is uncoded QPSK. The multiframe employed is indicated in Figure 2. Three precoding schemes are used: single-antenna, maximum ratio, and zero-forcing. In the maximum ratio case the weights are given by

$$
\mathbf{w}_{\mathrm{MR}}=c \hat{\mathbf{h}}_{\mathrm{d}} \text {, }
$$

where $c$ is a scalar and $\widehat{\mathbf{h}}_{\mathrm{d}}$ is the channel estimated from the uplink (the channels in this section are defined as the conjugate transpose of $\widetilde{\mathbf{H}}$ defined in Section 2). In the zeroforcing case the transmit vector is selected as

$$
\mathbf{w}_{\mathrm{ZF}}=c\left(\mathbf{I}-\frac{1}{\left\|\hat{\mathbf{h}}_{i}\right\|^{2}} \hat{\mathbf{h}}_{i} \hat{\mathbf{h}}_{i}^{*}\right) \mathbf{h}_{\mathrm{d}},
$$

where $\hat{\mathbf{h}}_{i}$ is the channel estimate of the cochannel user seen at the base-station. In the single antenna precoding case, which is included as a reference only, one element of the precoding vectors is set to zero. All precoders have the same norm. The precoding should ideally be performed on a subcarrier basis. However, our emulation strategy only allows one set of weights for all subcarriers as we will see below.

In the first frame calibration signals are sent internally from antenna no. 1 to antenna no. 2, while in the second the signal is sent in the opposite direction. The received signal is used as described in Section 2 in order to estimate the TDD calibration coefficient that is $\left(c_{2}^{\mathrm{tx}} c_{1}^{\mathrm{rx}}\right) /\left(c_{1}^{\mathrm{tx}} c_{2}^{\mathrm{rx}}\right)$. The calibration scheme is applied independently for each subcarrier using a CW signal with the corresponding frequency.

The uplink and downlink frames in Figure 2 are identical, except that the uplink frame is transmitted from the mobilestation to the base-station and the downlink frame in the opposite direction. The frames contain fourteen burst pairs. The two bursts in a burst pair are identical except that the first one is transmitted on antenna no. 1 and the other on antenna no. 2. Each burst contains fourteen OFDM symbols. There is a lot of space in all the $6 \mathrm{~ms}$ buffers. This space could be eliminated, but our interest is to study the principal limitations of TDD reciprocity-based precoding and not to optimise the throughput of our test system. In addition, the space is utilised in order to estimate the noise level. The transmitted OFDM signals are pre-calculated in Matlab, and the received signals are then stored on harddisc for postprocessing in Matlab. We are able to emulate the performance of a TDD reciprocity-based system with two base-stations and mobile-stations by combining multiple measurements. The details of this emulation are given in Appendix A. A key point in the emulation is the fact that we have transmitted the same burst with both antennas. This allows us to weight the contributions from the two antennas of the base-station and sum them to construct the signal that would have been received at the mobile-station given a certain precoder. This relies on the assumption that the receiver is linear, which appears to be a mild assumption.

In the emulation process, the uplink channels are estimated by the base-station based from the uplink frame; see Figure 2. The channel estimation is done independently among the subcarriers by cross-correlation with the transmitted signal. The base-station then applies the calibration coefficient to obtain estimates of the downlink channels. Given the downlink channel the base-station can calculate the precoding weights. The signal received at the mobilestation from the two base-stations is then calculated by 


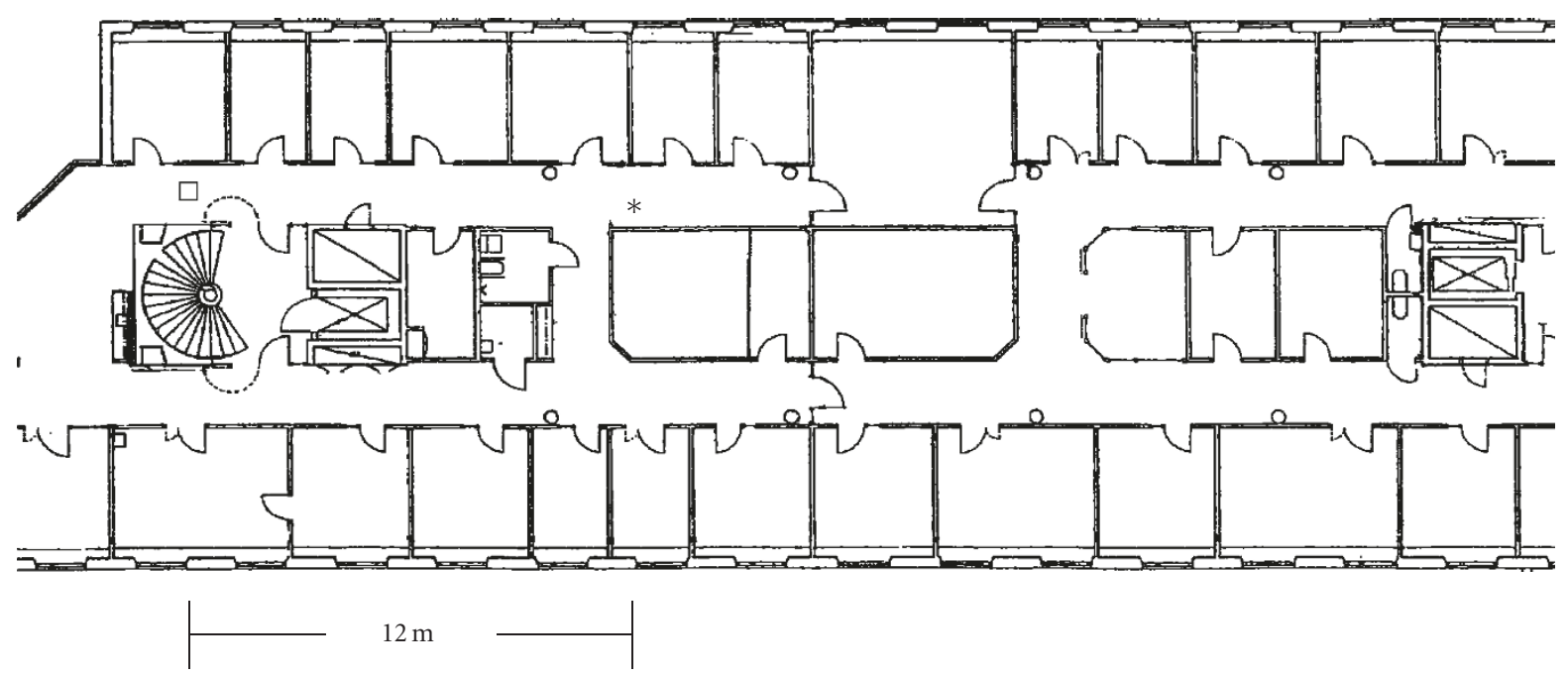

Figure 3: Floor-plan layout. The asterix and square indicate the postion of the two base-stations. The mobile station moved in and out of the offices between the two base-stations.

weighing the antenna signals according to the selected weights. The mobile-station then demodulates the combined signal assuming the first symbol to be known. More details are provided in Appendix A.

\section{Measurement Campaign}

The measurements were done on in an office environment. The base-station was placed at the points marked with an asterisk and a square (Figure 3 ). The mobile-station was moving at some $5-10 \mathrm{~cm} / \mathrm{sec}$ moving in and out of offices in between the base-stations. The multiframes were separated by some ten seconds to achieve fading decorrelation between measurements. Some measurements close to the base-station had to be removed because the receiver was saturated from the strong signal and the absence of automatic gain control. A total of 152 good multiframes were collected. These measurements are divided into four parts: A, B, C, and D. These parts represent the different paths in 152/4 $=38$ twobase-station two-mobile scenarios. This is described in more detail in Appendix A.

Dual slant polarised patch antennas are used as transmitter antennas and a single slant patch as receiver antenna. The output power is $-6 \mathrm{dBm}$ divided equally among the five carriers with $250 \mathrm{kHz}$ spacing. A higher output power leads to bit errors due to nonlinearity in the power amplifiers (varies between amplifier units). The carrier frequency is $1902.5 \mathrm{MHz}$.

\section{Measurement Results}

The performance of single-antenna, maximum-ratio, and zero-forcing pre-coding in a two-cell scenario is evaluated based on measurements as described in Appendix A. The mean bit error rate (uncoded) is $17.1 \%, 11.7 \%$, and $0.9 \%$, for single-antenna, maximum-ratio, and zero-forcing, respectively. The outage probability that is the probability of a

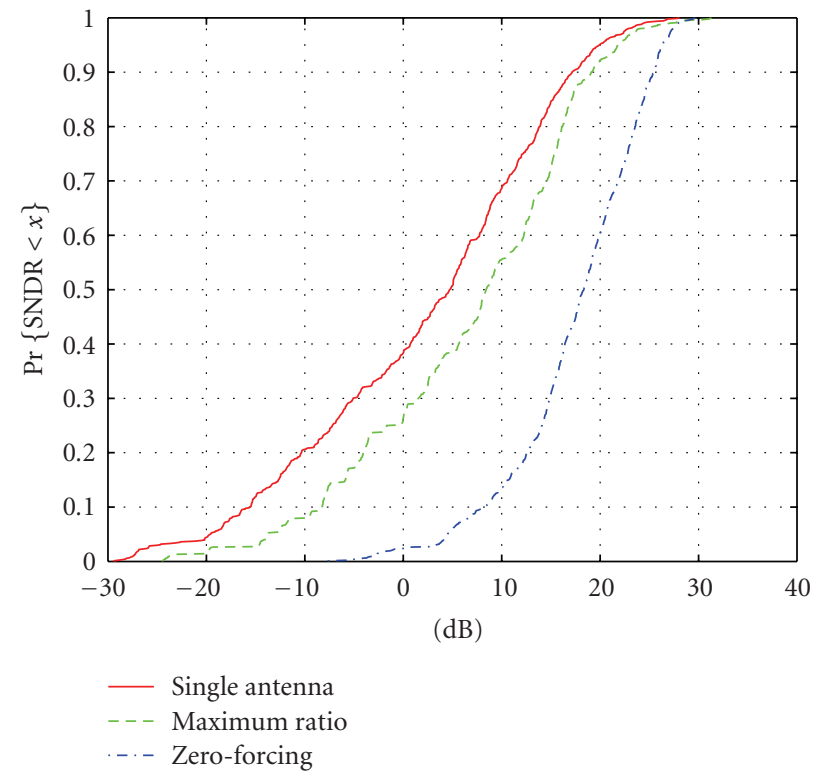

FIGURE 4: Cumulative distribution of SINDR at the receiver.

single bit error or more in a $6 \mathrm{~ms}$ frame is $74 \%, 39 \%$, and $14 \%$ for single-antenna, maximum-ratio, and zeroforcing, respectively. The cumulative distribution function of the obtained signal to interference, noise and distortion measurements are shown in Figure 4. We note that the zero-forcing outperforms single-antenna and maximumratio transmission, where the difference between the latter two techniques is relatively minor. The system is clearly interference limited as the signal to noise ratio was found to be higher than $30 \mathrm{~dB}, 95 \%$ of the time.

5.1. Coordinated Multipoint Scenario (CoMP). We now consider a coordinated multipoint (CoMP) scenario where scheduling is performed jointly for the two cells. We further 


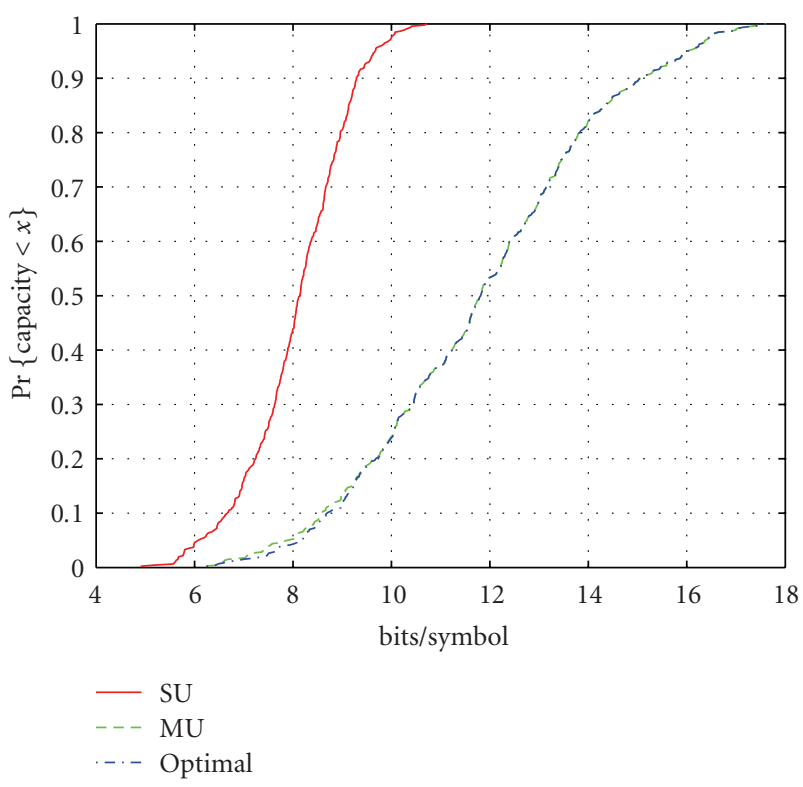

Figure 5: Throughput of single-user and multiuser scheduling.

assume that adaptive modulation and coding are employed, and that the sum of interference noise and distortion can be modelled as Gaussian noise. Under these assumptions we model the throughput of each channel use as $\log _{2}(1+$ SNIDR) (a channel is here a certain subcarrier and a certain OFDM symbol). Two users are considered as before. A joint scheduler would in such a scenario select the best way of sharing the channel, either a single-user at a time (SU) that is, using time division or by simultaneous use by both users (MU) (in the SU case we use maximum-ratio transmission while we use zero-forcing in the MU case). However, as we will see later in Section 6.3, predicting the SNIDR is difficult which makes it difficult to realise these capacities in practise. Here, however we assume the genie aided scenario where the base-stations knows exactly the SNIDR. Thus for each of the 38 measurements we select the solution that gives the maximum sum capacity. In the SU case we of course multiply the single-user capacity by a factor 0.5 to account for the time division sharing of the channel. Figure 5 shows the cumulative distribution of the optimum solution together with the reference cases of TD-only and SU-only. In the optimal mix, the MU-solution is chosen in $95 \%$ of the cases which shows that zero-forcing is meaningful.

5.2. Ideal Case versus the Measurements. A natural question is how far from ideal theory the measurement results herein are. The ideal case represents the way most researchers would simulate the system considered, namely, assuming perfect channel knowledge at the base-stations and only thermal noise and cochannel interference impairing the reception. The performance of this case has been obtained for zeroforcing and is labelled as "ideal" in Figure 7 (more details are given in the next section). In contrast the curve labelled "measurement" represents the results actually obtained from the measurements. As is evident to the reader, the gap

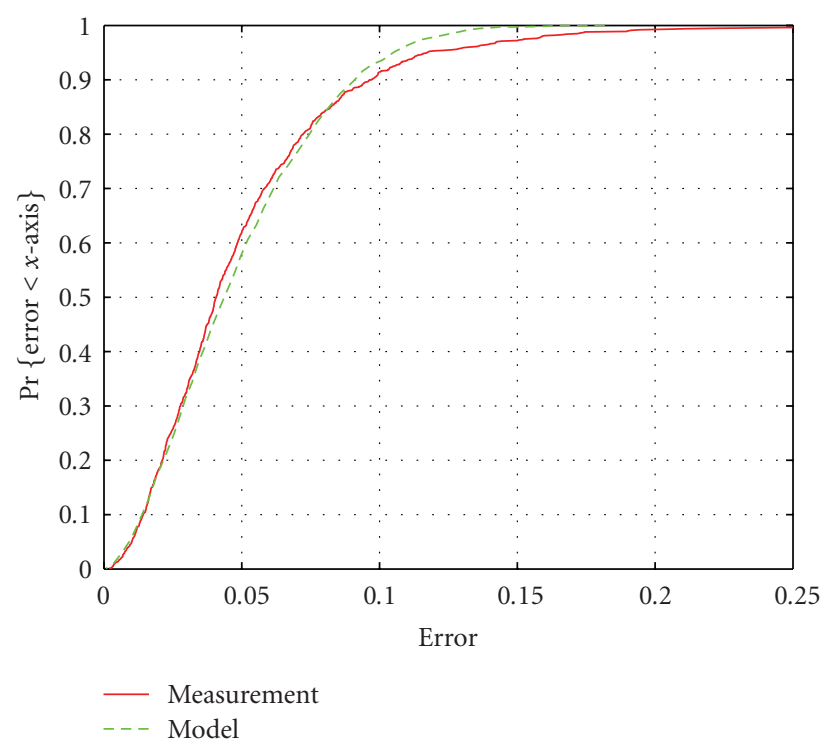

FIGURE 6: Measured channel prediction error.

between the two curves is substantial. This issue is analysed further in the next section.

\section{Analysis of Impairments}

In this section we analyse the impact of radio frequency (RF) impairments on the performance of the system.

6.1. Errors in the Channel Prediction. The "prediction" of the downlink channel is obtained by calculating the calibration factor $\left(c_{2}^{\mathrm{tx}} c_{1}^{\mathrm{rx}}\right) /\left(c_{1}^{\mathrm{tx}} c_{2}^{\mathrm{rx}}\right)$ and applying it to the uplink channel estimate according to (6). Based on our measurements we can compare the error between the "predicted" downlink channel estimate and the true channel estimate. In this comparison we use the downlink channel estimated at the mobile-station as the "true" downlink channel. We define the error, $e$, between the "predicted" downlink channel $\widetilde{\mathbf{h}}$ and the "true" downlink channel $\mathbf{h}_{\mathrm{DL}}$ as

$$
e=\sqrt{1-\frac{\left|\tilde{\mathbf{h}}^{*} \mathbf{h}_{\mathrm{DL}}\right|^{2}}{\|\tilde{\mathbf{h}}\|^{2}\left\|\mathbf{h}_{\mathrm{DL}}\right\|^{2}}}
$$

We note that this definition is invariant to any scaling error. The error is also related to the performance of zeroforcing precoding as described in Appendix B. In Figure 6 the cumulative distribution of the prediction error, $e$, is plotted as the curve with legend "measurement." It has been verified that the influence of noise is negligible in these measurements. Also plotted in Figure 6 is a "model" curve which is the error, $e$, obtained from simulations using the following error model:

$$
\tilde{\mathbf{h}}_{\text {model }}=\mathbf{h}_{\mathrm{DL}}+\mathbf{e}
$$




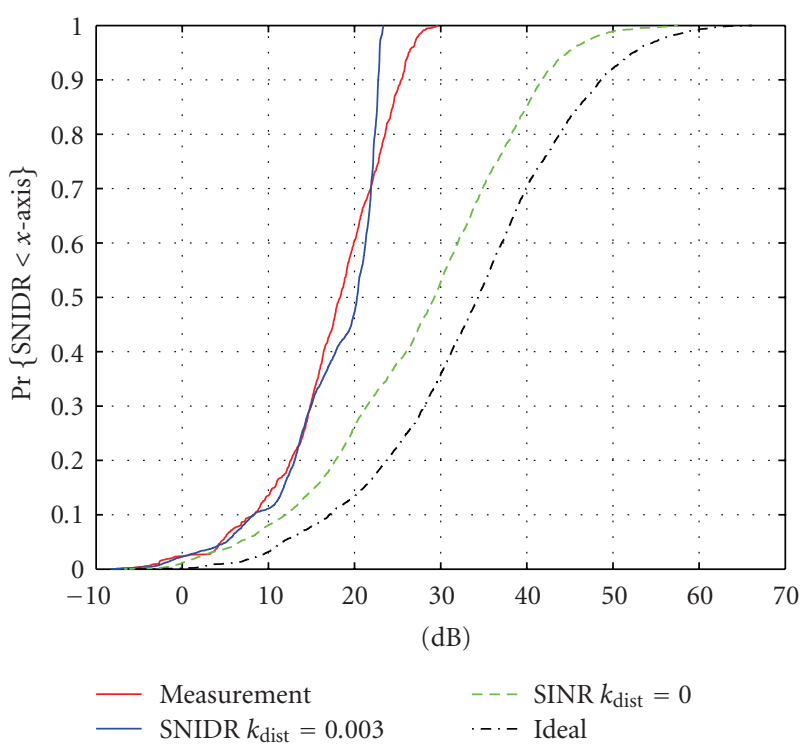

FIGURE 7: Cumulative distribution of SNIDR at the receiver.

where $\mathbf{e}$ is a complex Gaussian random vector with independent elements. The covariance matrix of $e$ is given by

$$
E\left\{\mathbf{e e}^{*}\right\}=k_{\text {est }} \operatorname{diag}\left(\mathbf{h}_{\mathrm{DL}} \mathbf{h}_{\mathrm{DL}}^{*}\right),
$$

where $k_{\text {est }}=0.01$. The plot shows fair agreement between the model and the measurements. This does not fully prove the model since the model is multidimensional while the error measure is scalar. The intuition for the model is that errors are multiplicative and thus proportional to the channel amplitude.

6.2. Influence of Distortion. In Section 5 we presented the performance of the system in terms of bit error rate (BER) and signal to interference noise and distortion (SNIDR). In order to investigate the contribution from distortion to the SNIDR distribution of the zero-forcing solution, the SNIDR obtained from the measurements is shown in Figure 7 as the curve labelled "measurement." Also shown in the figure is a curve labelled "SINR, $k_{\text {dist }}=0$." This curve was obtained by using the exact same precoder weights as in the "measurement" curve. However, we here calculate the signal to noise and interference ratio (SINR), according to

$$
\operatorname{SINR}=\frac{\left\|\mathbf{w}_{\mathrm{d}}^{*} \mathbf{h}_{\mathrm{d}}^{\mathrm{DL}}\right\|^{2}}{\left\|\mathbf{w}_{\mathrm{c}}^{*} \mathbf{h}_{\mathrm{c}}^{\mathrm{DL}}\right\|^{2}+\sigma_{\mathrm{n}}^{2}},
$$

where the subscripts "d" and " $\mathrm{c}$ " denote entities associated with the desired and interfering (i.e. cochannel) base-station, respectively. The noise level is based on measurements during periods when there is no signal present. The channels used in (13) are based on measurements from the data at the mobile-station, while the weighting vectors were based on the downlink channels predicted from the uplink data. The gap between the "measurement" and "SINR, $k_{\text {dist }}=0$ " curve represents the influence of distortion.
In a typical simulation of our system one would assume that the channel estimation is perfect (the noise level is very small in our measurements). The performance of such system is described by the curve "ideal" in Figure 7, where we have used the channel matrices estimated in downlink when calculating the pre-coding weights, when applying (13). Thus the gap between the "measurement" and "ideal" represents the total gap between theory and practise. In order to bridge this gap we have presented an empirical model for the channel prediction error in Section 6.1. However, the distortion is also responsible for a great portion of the gap between the "ideal" and "measurement" curves. Among the major contributors of distortions in OFDM systems are nonlinear amplifiers and phase-noise [10]. Previous studies suggest that these can be modelled as Gaussian $[10,12,14]$. Thus with each transmitter or receiver branch we associate a Gaussian noise to represent the distortion. We select the power of this noise to be proportional to the transmitted signal. Considering phase-noise this assumption can be motivated from theory (see (16) of [10]), while for amplifier nonlinearities it is only approximate. Thus the power of the distortion noise in each receiver or transmitter branch is assumed to be given by

$$
\sigma_{\mathrm{d}}^{2}=k_{\text {dist }} P,
$$

where the factor $k_{\text {dist }}$ can be interpreted as the error-vectormagnitude [15]. We assume that the distortion noise is independent between transmitter branches as was shown by measurements in [12] and assumed in [11]. We further assume that the distortion in the transmitter and receiver chains are of equal power (i.e., the same $k_{\text {dist }}$ coefficient applies) since we are not able to separate them in our measurements. Since the power of the signal at the transmitter is given by the weights of the corresponding antenna, the transmitter noise will be white with a covariance matrix given by $k_{\text {dist }} \operatorname{diag}\left(\left|w_{1}\right|^{2}, \ldots,\left|w_{m}\right|^{2}\right)$, where $w_{1}, \ldots, w_{m}$ are the transmitter weights. This transmitter noise then passes through the channel $\mathbf{h}^{\mathrm{DL}}$. The power of transmitter distortion is obtained by weighting the contribution of the transmitter antennas with the channel gains and summing the result. In compact form we can write this resulting noise power as $\left\|\mathbf{w} \odot \mathbf{h}^{\mathrm{DL}}\right\|^{2}$. The distortion noise of the receiver is simply obtained by taking the power of the received signal and multiplying by $k_{\text {dist }}$. By applying these principles to our case of two base-stations and a single mobile antenna we obtain

$$
\operatorname{SINDR}=\frac{\left\|\mathbf{w}_{\mathrm{d}}^{*} \mathbf{h}_{\mathrm{d}}^{\mathrm{DL}}\right\|^{2}}{\left\|\mathbf{w}_{\mathrm{c}}^{*} \mathbf{h}_{\mathrm{c}}^{\mathrm{DL}}\right\|^{2}+\sigma_{\text {tot }}^{2}},
$$

where $\sigma_{\text {tot }}^{2}$ is given by

$$
\begin{gathered}
\sigma_{\text {tot }}^{2}=\sigma_{n}^{2}+k_{\text {dist }}\left(\left\|\mathbf{w}_{\mathrm{d}} \odot \mathbf{h}_{\mathrm{d}}^{\mathrm{DL}}\right\|^{2}+\left\|\mathbf{w}_{\mathrm{c}} \odot \mathbf{h}_{\mathrm{c}}^{\mathrm{DL}}\right\|^{2}+\left|\mathbf{w}_{\mathrm{d}}^{*} \mathbf{h}_{\mathrm{d}}^{\mathrm{DL}}\right|^{2}\right. \\
\left.+\left|\mathbf{w}_{\mathrm{c}}^{*} \mathbf{h}_{\mathrm{c}}^{\mathrm{DL}}\right|^{2}\right) .
\end{gathered}
$$




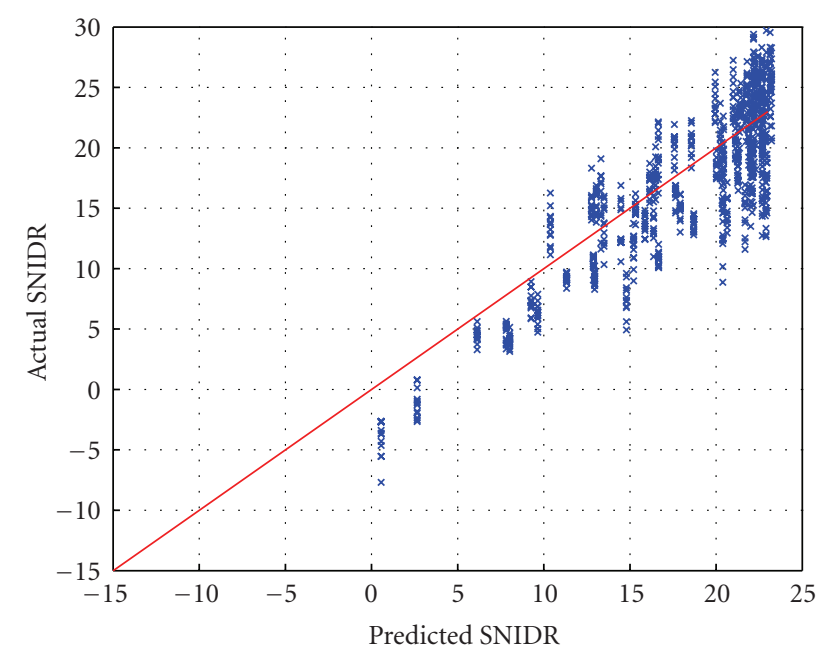

FIgure 8: Prediction of the actual SNIDR. The $x$-axis is the prediction and the $y$-axis the actually measured SNIDR.

In order to obtain a value of $k_{\text {dist }}$ we first conducted a series of measurements using a single transmitter antenna measurement. Based on these measurements we set $k_{\text {dist }}=$ 0.003 . The SNIDR calculated by using (15) is shown in Figure 7 . The curve is fairly close to the measurement results up to the $80 \%$ level of the CDF.

6.3. Predicting the Performance. In order to be able to do CoMP as described in Section 5.1. we need to be able to predict the downlink SNIDR, in order to do scheduling and select the appropriate modulation and coding scheme. In attempt to predict the downlink SNIDR we use (15). However, now we use the predicted downlink channels instead of the true downlink channels when evaluating (16) since the base-station does not access to the true downlink channel. The results are shown in Figure 8 where each point represents a measurement result. The $x$-axis of the point is the SNIDR predicted from (5) and the $y$-axis the actual measured SNIDR. The prediction is relatively good in average but the standard deviation of the prediction error is $3.5 \mathrm{~dB}$. It is obvious that a better SNIDR prediction would be much desired. Therefore, more research into this area is needed.

\section{Conclusions}

In this paper we presented a method for TDD calibration based on the reciprocity principle. The method is based on transmitting and receiving signals between the elements of the antenna array. The method does not require interactions with other nodes or additional calibration circuitry. How to use the method in a MIMO context is also indicated. We further describe an implementation of maximum-ratio and zero-forcing precoding on a wireless testbed called USRP (see http://www.ettus.com/). We study the performance in terms of bit-error rate (BER) signal to noise, interference, and distortion ratio (SNIDR) and throughput. The use of zero-forcing precoder is shown to outperform maximum ratio transmission.

We also analyse the error in the downlink channel prediction by comparing the predicted channel vectors with those actually obtained at the mobile station. A model for the prediction error based on the measurements is proposed. The impact of distortion is also factored out from the measurements. We show that a simple error vector model provides a reasonable model for the errors in an average sense.

However, when we try to predict the SNIDR such as required in a coordinated multipoint scenario (CoMP) with joint scheduling, the prediction error is substantial (standard deviation $3.7 \mathrm{~dB}$ ). This shows that there is room for substantial improvement in this respect.

\section{Appendices}

\section{A. Details of the Implementation on USRP}

A system with a single base-station and mobile-station can be emulated as follows.

(1) Calculate the calibration constant $\left(c_{2}^{\mathrm{tx}} c_{1}^{\mathrm{rx}}\right) /\left(c_{1}^{\mathrm{tx}} c_{2}^{\mathrm{rx}}\right)$ from the calibration data stored at the base-station.

(2) Estimate the uplink channel based on the data stored at the base-station.

(3) Predict the downlink channel using the uplink data and the estimated calibration data.

(4) Calculate the precoder based on the obtained channel knowledge.

(5) Construct the signal received by the mobile-station by adding and weighting the two parts of the burst pairs using the previously obtained weights.

(6) Demodulate the received signal assuming that the first OFDM symbol of the burst is known.

(7) Estimate the SINDR by calculating the mean square error between the sample-points and the true constellation points.

There is one problem with the enumeration above. In an OFDM system we would ideally transmit with different precoder weights on different subcarriers. However, the above emulation scheme does not allow that. On the other hand, in our indoor propagation scenario the channel can be regarded as flat over the five subcarriers spanning $1.25 \mathrm{MHz}$, and thus the loss is negligible. Note, however, that we are still able to study the channel estimation error on all the subcarriers.

In order to develop the emulation scheme above for a case with two base- and mobile-stations we need to elaborate the procedure further. In order to do so, we need first to describe the USRP measurement campaign in detail. The campaign was done in an office floor at a speed of $5-10 \mathrm{~cm} / \mathrm{sec}$ with ten seconds between multiframes to decorrelation in the fast fading. The USRP measurement campaign consists of four parts, campaign $\mathrm{A}, \mathrm{B}, \mathrm{C}$, and $\mathrm{D}$. In campaign $\mathrm{A}$ and $\mathrm{B}$ the 
base-station was positioned at the asterisk of Figure 3 while it was positioned at the square in campaign $\mathrm{C}$ and $\mathrm{D}$. The mobile-station was typically in the corridor and office rooms close to the base-station marked by an asterisk in campaign $\mathrm{A}$ and $\mathrm{D}$, while it was close to the base-station marked by a square in campaign $\mathrm{B}$ and $\mathrm{C}$. In each subcampaign 38 measurements were made. We use the data measured in campaign $\mathrm{A}$ and $\mathrm{D}$ to represent the channel between user no. 1 and base-station no. 1 and no. 2, respectively, while the data measured in campaign $\mathrm{B}$ and $\mathrm{C}$ represents the channel between mobile-station no. 2 and base-station no. 1 and no. 2, respectively. The performance of a two base-station two mobile-station is then done by repeating the following procedure for the 38 measured quartets of multiframes.

(1) Calculate the calibration constant $\left(c_{2}^{\mathrm{tx}} c_{1}^{\mathrm{rx}}\right) /\left(c_{1}^{\mathrm{tx}} c_{2}^{\mathrm{rx}}\right)$ for base-station no. 1 using data from campaign $\mathrm{A}$ and D.

(2) Do likewise for base-station no. 2.

(3) Estimate the uplink channels between base-station no. 1 and mobile-station no. 1 using calibration and uplink data from campaign A.

(4) Estimate the uplink channels between base-station no. 1 and mobile-station no. 2 using calibration and uplink data from campaign D.

(5) Do likewise for base-station no. 2 using data from campaign $\mathrm{B}$ and $\mathrm{C}$.

(6) Calculate the precoders for base-station no. 1 and no. 2.

(7) Construct the signal received at mobile-station no. 1 by adding the contribution from base-station no. 1 and no. 2 using data from campaign A and D. The contribution from base-station no. 1 is the sum of the two transmitter antennas weighted by the precoder of that base-station and likewise for base-station no. 2 . The signal from base-station no. 2 is offset one burst pair so that the interfering signal carries a different information content than the desired signal.

(8) Demodulate the signal received at mobile-station no. 1. The first OFDM symbol of the desired basestation is assumed known. The interference (i.e., the contribution from the other base-station) is removed from the training OFDM symbol.

(9) Estimate the SINDR by calculating the mean square error between the sample-points and the true constellation points.

(10) Repeat step (5)-(7) for mobile-station no. 2 with obvious changes.

Note that we remove the interference from the channel estimation, and no interference is added to the uplink measurements.

During the measurements the nodes were synchronised using a cable. The cable is connected to a general purpose pin on each of the USRPs. The two nodes are polling the pin continuously. When the pin changes polarity a frame is started. The cable is driven by a square-wave generator with half-period of $6 \mathrm{~ms}$. Due to latencies in the USRP, USB, and PCs the useful signal appears 1-2 ms into the received buffer. The latency varies from frame to frame. Each frame starts with a synchronisation sequence of 100 samples. When the data is processed the timing of the received burst is obtained by cross-correlating the received signal with the known synchronisation sequence. This correlation is done with several frequency offsets to simultaneously obtain the frequency offset.

\section{B. The Chosen Error Measure}

Let us divide the true downlink channel $\mathbf{h}_{\mathrm{DL}}$ into two parts, one which is aligned with the channel estimate, $\tilde{\mathbf{h}}$, and one which is orthogonal to this channel estimate, that is,

$$
\begin{aligned}
\mathbf{h}_{\mathrm{DL}} & =\mathbf{P}_{\widetilde{\mathbf{h}}} \mathbf{h}_{\mathrm{DL}}+\mathbf{P}_{\widetilde{\mathbf{h}}}^{\perp} \mathbf{h}_{\mathrm{DL}} \\
& =\frac{\widetilde{\mathbf{h}}^{*} \mathbf{h}_{\mathrm{DL}}}{\| \tilde{\mathbf{h}}+\overline{\mathbf{e}} .}
\end{aligned}
$$

The "power" of the error vector is given by

$$
\begin{aligned}
\|\overline{\mathbf{e}}\|^{2} & =\mathbf{h}_{\mathrm{DL}}^{*} \mathbf{P}_{\tilde{\mathrm{h}}}^{\perp} \mathbf{h}_{\mathrm{DL}} \\
& =\left\|\mathbf{h}_{\mathrm{DL}}\right\|^{2}-\frac{\left\|\mathbf{h}_{\mathrm{DL}}^{*} \tilde{\mathbf{h}}\right\|^{2}}{\|\tilde{\mathbf{h}}\|^{2}} .
\end{aligned}
$$

If the channel estimate $\tilde{\mathbf{h}}$ is that of a cochannel user, a zero-forcing precoder would choose a weighting such that $\mathbf{w}_{\mathrm{ZF}}^{*} \tilde{\mathbf{h}}=0$. The remaining interference is then given by $\left|\mathbf{w}_{Z \mathrm{~F}}^{*} \overline{\mathbf{e}}\right|^{2}$, where the power of $\overline{\mathbf{e}}$ is given by (B.2). The power of $\overline{\mathbf{e}}$ needs to be set in relation to $\mathbf{h}_{\mathrm{DL}}$. We therefore chose to divide the power of $\overline{\mathbf{e}}$ by the power of $\mathbf{h}_{\mathrm{DL}}$ thus obtaining

$$
\begin{aligned}
\frac{\|\overline{\mathbf{e}}\|^{2}}{\left\|\mathbf{h}_{\mathrm{DL}}\right\|^{2}} & =1-\frac{\left\|\mathbf{h}_{\mathrm{DL}}^{*} \tilde{\mathbf{h}}\right\|^{2}}{\|\tilde{\mathbf{h}}\|^{2}\left\|\mathbf{h}_{\mathrm{DL}}\right\|^{2}} \\
& =e^{2},
\end{aligned}
$$

that is, the square of our chosen error measure $e$.

\section{Acknowledgments}

The research leading to these results has received funding from the European Research Council under the European Community's Seventh Framework Programme (FP7/20072013)/ERC Grant agreement no. 228044. The work has also been performed partly within the framework of the European Commission funded IST-2002-2.3.4.1 COOPCOM project. 


\section{References}

[1] M. Bengtsson and B. Ottersten, "Optimal and suboptimal transmit beamforming," in Handbook of Antennas in Wireless Communications, L. C. Godara, Ed., CRC Press, 2001.

[2] D. P. Palomar, J. M. Cioffi, and M. A. Lagunas, "Joint Tx-Rx beamforming design for multicarrier MIMO channels: a unified framework for convex optimization," IEEE Transactions on Signal Processing, vol. 51, no. 9, pp. 2381-2401, 2003.

[3] Q. H. Spencer, A. L. Swindlehurst, and M. Haardt, "Zeroforcing methods for downlink spatial multiplexing in multiuser MIMO channels," IEEE Transactions on Signal Processing, vol. 52, no. 2, pp. 461-471, 2004.

[4] S. Ramo, J. R. Whinnery, and T. Van Duzer, Fields and Waves in Communication Electronics, John Wiley \& Sons, New York, NY, USA, 3rd edition, 1993.

[5] J. H. Winters, "Optimum combining in digital mobile radio with cochannel interference," IEEE Transactions on Vehicular Technology, vol. 33, no. 3, pp. 144-155, 1984.

[6] S. Gollakota, S. D. Perli, and D. Katabi, "Interference alignment and cancellation," in Proceedings of the ACM Conference on Data Communication (SIGCOMM '09), pp. 159-170, August 2009.

[7] J.-C. Guey and L. D. Larsson, "Modeling and evaluation of MIMO systems exploiting channel reciprocity in TDD mode," in Proceedings of the IEEE Vehicular Technology Conference, vol. 6, pp. 4265-4269, September 2004.

[8] M. Guillaud, D. T. M. Slock, and R. Knopp, "A practical method for wireless channel reciprocity exploitation through relative calibration," in Proceedings of the 8th International Symposium on Signal Processing and Its Applications (ISSPA '05), pp. 403-406, August 2005.

[9] K. Nishimori, K. Cho, Y. Takatori, and T. Hori, "Automatic calibration method using transmitting signals of an adaptive array for TDD systems," IEEE Transactions on Vehicular Technology, vol. 50, no. 6, pp. 1636-1640, 2001.

[10] R. Corvaja, E. Costa, and S. Pupolin, "Analysis of M-QAMOFDM transmission system performance in the presence of phase noise and nonlinear amplifiers," in Proceedings of the 28th European Microwave Conference, vol. 1, pp. 481-486, October 1998.

[11] B. Göransson, S. Grant, E. Larsson, and Z. Feng, "Effect of transmitter and receiver impairments on the performance of MIMO in HSDPA," in Proceedings of the 9th IEEE Workshop on Signal Processing Advanced in Wireless Communications, pp. 496-500, 2008.

[12] C. Studer, M. Wenk, and A. Burg, "MIMO transmission with residual transmit-RF impairments," in Proceedings of the International ITG Workshop on Smart Antennas (WSA '10), pp. 189-196, February 2010.

[13] E. Blossom, "GNU Radio: tools for exploring the radio frequency spectrum," Linux Journal, vol. 2004, no. 122, 2004.

[14] D. Dardari, V. Tralli, and A. Vaccari, "A theoretical characterization of nonlinear distortion effects in ofdm systems," IEEE Transactions on Communications, vol. 48, no. 10, pp. 1755$1764,2000$.

[15] R. A. Shafik, M. S. Rahman, A. H. M. R. Islam, and N. S. Ashraf, "On the error vector magnitude as a performance metric and comparative analysis," in Proceedings of the IEEE-ICET International Conference on Emerging Technologies, Pershawar, Pakistan, November 2006. 